\title{
Organic solar cells based on non-fullerene acceptors
}

Jianhui Hou, Olle Inganäs, Richard H. Friend and Feng Gao

The self-archived postprint version of this journal article is available at Linköping University Institutional Repository (DiVA):

http://urn.kb.se/resolve?urn=urn:nbn:se:liu:diva-144871

N.B.: When citing this work, cite the original publication.

Hou, J., Inganäs, O., Friend, R. H., Gao, F., (2018), Organic solar cells based on non-fullerene acceptors, Nature Materials, 17(2), 119-128. https://doi.org/10.1038/NMAT5063

Original publication available at:

https://doi.org/10.1038/NMAT5063

Copyright: Nature Publishing Group

http://www.nature.com/ 


\title{
Organic solar cells based on non-fullerene acceptors
}

\author{
Jianhui Hou ${ }^{1}$, Olle Inganäs ${ }^{2}$, Richard H. Friend ${ }^{3}$, Feng Gao ${ }^{2}$ \\ ${ }^{1}$ Beijing National Laboratory for Molecular Sciences, State Key Laboratory of Polymer Physics and Chemistry, \\ Institute of Chemistry, Chinese Academy of Sciences, Beijing 100190, China \\ ${ }^{2}$ Biomolecular and organic electronics, Department of Physics, Chemistry and Biology (IFM), Linköping \\ University, Linköping SE-58183, Sweden \\ ${ }^{3}$ Cavendish Laboratory, J J Thomson Avenue, Cambridge CB3 OHE, United Kingdom
}

\begin{abstract}
Organic solar cells (OSCs) have been dominated by donor:acceptor blends based on fullerene acceptors for over two decades. This situation has changed very recently, with non-fullerene (NF) OSCs developing very quickly. The power conversion efficiencies of NF OSCs have now reached a value of over $13 \%$, which is higher than the best fullerenebased OSCs. NF acceptors show great tunability in absorption spectra and electron energy levels, providing a wide range of new opportunities. The co-existence of low voltage losses and high current generation indicates that new regimes of device physics and photophysics are reached in these systems. This Review highlights these opportunities made possible by NF acceptors, and also discuss the challenges facing the development of NF OSCs for practical applications.
\end{abstract}


Light absorption in organic semiconductors generates strongly bound excitons. Donor (D): acceptor (A) bulk-heterojunction (BHJ) structures, suitable for low-cost solution processing, provide an efficient approach to split the excitons into free carriers. ${ }^{1,2}$ Among different acceptor materials, fullerene derivatives attracted the most attention and gave the highest power conversion efficiencies (PCEs) for almost two decades. Unique to fullerene derivatives is their ball-like fully conjugated structure, which provides strong electron-accepting and isotropic electron-transport capabilities and facilitates electron delocalization at the D:A interfaces. ${ }^{3}$ As such, fullerene derivatives were believed to be a critical component for efficient operation of organic solar cells (OSCs). Indeed, acceptor materials based on molecules other than fullerene derivatives, generally categorized as non-fullerene (NF) acceptors, usually resulted in low PCEs, ${ }^{1,4}$ which were mainly attributed to the difficulties in the morphological control. ${ }^{5}$

However, this situation has changed recently, with quick development of NF OSCs. The PCEs of NF OSCs have increased dramatically since 2015, now reaching a high value of $13.1 \%{ }^{6}$ Such a high value is better than $11.7 \%$ in the best fullerene-based OSCs. ${ }^{7,8}$ The quick development of NF OSCs during the past two years has benefited a lot from the synthetic methods, materials design strategies and device engineering protocols developed during the past two decades for fullerene-based OSCs. The wide range of donor molecules developed for fullerene-based OSCs provides a rich library for immediate use in NF OSCs. In addition, various design strategies originally developed for donor molecules are readily available to tune the absorption spectra and energy levels of NF acceptors, allowing better flexibility in realizing donor-acceptor systems with complementary absorption and optimized energy band diagram. The development of a few high-performing molecules, discussed in the next section, has also contributed to attracting the interest of the research community on NF OSCs.

From the device point of view, a feature that contributes to high PCEs in NF OSCs is that excitons can separate efficiently upon negligible driving energies. ${ }^{9-14}$ As a result, NF OSCs often show high photocurrent and low voltage losses at the same time. In contrast, charge separation in fullerene-based OSCs usually becomes problematic under low driving energies, presenting a trade-off between high photocurrent and high photovoltage. ${ }^{15,16}$ Currently, the device physics and photo-physics investigations are lagging behind the rapid developments of 
materials and device engineering. A fundamental question open to the community is how the excitons split into free carriers in these NF OSCs with low driving energies.

Along with opportunities, one of the key challenges for NF acceptors might lie in their anisotropic structures. It is well acknowledged that D:A $\pi-\pi$ interactions, which depend on the molecular orientation between the donor and acceptor materials, are very important for the charge transfer and transport in the devices. ${ }^{17}$ Compared with the isotropic ball-like conjugated backbones in fullerene derivatives, the anisotropic conjugated structures of NF acceptors make it more challenging to ensure efficient $\pi-\pi$ interactions. ${ }^{18-21}$ Therefore, it becomes critically important to pair the donors with right acceptors in NF OSCs. From this aspect, the great diversity in chemical structures of NF acceptors also brings challenges for morphological control in devices, not only due to the well-known requirement for fine-tuning the phase separation but also because of the demand for molecular orientation control.

The development of NF acceptors presents opportunities which are otherwise not possible in fullerene-based OSCs, and also opens up challenges which are waiting to be tackled for future applications of this promising technology. In addition, the operation of NF OSCs also implies new working mechanisms, which could be fundamentally different from those in fullerenebased OSCs. This Review aims to discuss these opportunities, challenges, and working mechanisms, hoping to foster further advances in this field. The PCEs of NF OSCs are promising for future improvement, through both materials chemistry and device engineering. As such, NF OSCs provide a promising technology for practical applications in the near future, especially considering that they have also shown excellent thermal stability. ${ }^{22,23}$

\section{State-of-the-art non-fullerene acceptors}

Based on chemical structures, state-of-the-art NF acceptors can be categorized into two types: acceptors based on fused aromatic diimides, and acceptors based on strong intramolecular electron push-pulling effects. These high-performance NF acceptors share two features in common (Figure 1a). First, their conjugated backbones are modified with $\pi$ conjugated functional groups involving highly electronegative elements, e.g. oxygen (in the form of a carbonyl group) and/ or nitrogen (in the forms of a cyano group or nitrogen-containing 
hetero-aromatic segments). Second, $\pi$-electrons in these functional groups can be well delocalized into the backbones. The first feature provides strong electron accepting abilities, and the second feature ensures a relatively low reorganization energy so that the accepted electrons can be transported easily without being trapped. In addition, for solution-processed OSCs, appropriate functional groups also need to be carefully designed to satisfy the solubility requirement - this is different from vacuum-deposited OSCs. We note that in vacuumdeposited solar cells, NF acceptors also demonstrate great potential in enhancing light absorption and hence attract considerable attention, ${ }^{24,25}$ consistent with recent development in solution-processed OSCs.

\section{Acceptors based on fused aromatic diimides}

Fused aromatic diimide derivative was the first acceptor material used in heterojunction OSCs - it was used as the electron acceptor in the pioneering bilayer devices in 1986 (Figure 1a). ${ }^{26}$ Since then, this type of materials have been considered as promising candidates for NF OSCs, and have attracted continuous attention. Among various derivatives of fused aromatic diimides, perylene diimides (PDIs) and naphthalene diimides (NDIs) (Figure 1b), widely used in the traditional dye industry, are two of the most intensively studied acceptor molecules because of their advantages in strong light absorption, low synthesis cost, and excellent stability.

PDIs have been frequently used in small molecular acceptors. ${ }^{27}$ The key considerations for the molecular design of PDI-based acceptors are to restrain their aggregation effects and improve their miscibility with polymer donors. PDIs have rigid and planar conjugated backbones. As a result, the PDI derivatives in solid states tend to form large-sized crystals, which are undesirable for the nanoscale morphology in the BHJ structures. ${ }^{4}$ In addition to the universal approaches (for instance, side chain engineering and solvent engineering) for controlling the aggregation effects, an effective method to solving this problem is to link PDI units, forming dimeric or multilinked PDIs (Figure 1b). ${ }^{28-30}$ These linked PDI derivatives are twisted, helping to break the aggregations of PDIs. For example, an efficient OSC based on a PDI derivative was obtained by using a dimer named as SF-PDI 2 , where two N-alkyl-substituted PDIs were linked by an unsubstituted spirofluorene (Figure 1c). ${ }^{9}$ 
In addition to small molecules, the polymers based on fused aromatic diimides (for instance, $\mathrm{N}$-alkyl-substituted PDIs and NDIs) have also been employed as effective acceptors in all polymer OSCs. ${ }^{31-34}$ The linkers also play a critical role in the design of these polymer acceptors. In this case, they not only help to modulate the aggregations but also provide additional opportunities to decrease the bandgap due to their extended conjugation. As a result, the polymer acceptors based on fused aromatic diimide derivatives often demonstrate low bandgaps. For example, N2200 (Figure 1d), which is a popular polymer acceptor based on NDI and bithiophene, has a low band gap of $1.46 \mathrm{eV}$. Its absorption spectrum and energy levels match very well with a range of wide and mid bandgap polymer donors originally developed for fullerene-based OSCs. Up to now, high PCEs around 9\% have been obtained for all polymer OSCs based on $\mathrm{N} 2200 .^{34,35}$

In spite of these progresses, the applications of fused aromatic diimide-based polymer acceptors in OSCs have critical challenges to be tackled. For example, there is a delicate balance between good solubility and effective interchain $\pi-\pi$ interactions for PDI-based polymer acceptors. Highly twisting linkers help to provide good solubility, but prevent effective interchain $\pi-\pi$ interactions, unfavorable for intermolecular charge transfer and transport. As a result, the linkers with decreased twisting effects are often used in these polymeric acceptors. In this case, long and branched alkyls, which offer sufficient solvation, have to be employed as the functional groups in order to ensure good solubility. However, these long branched alkyls are optoelectronically inert. This delicate requirement limits the development of suitable polymer acceptors. N2200 makes a good balance between high solubility and strong $\pi-\pi$ interactions, and hence becomes the most widely used polymeric acceptor in NF OSCs. In addition, when two polymers are mixed to form the BHJ structure, it is a great challenge to simultaneously realize small phase separation and avoid interchain entanglement between two different polymers from the aspect of polymer physics. This provides an additional obstacle that hampers the development of all polymer NF OSCs. As subtle changes in chemical structures could significantly affect the delicate balance and hence the device performance, systematic investigations on the molecular structures have to be carried out to further improve the performance of all polymer OSCs. 


\section{Acceptors based on strong intramolecular electron push-pulling effects}

The first acceptor in this category, CN-PPV (Figure 1a), was reported by the Cambridge group in their pioneering work on bulk heterojunction OSCs. ${ }^{1}$ As the cyano group has high electron negativity and is linked with the ethylene segment, accepted electrons can delocalize effectively along the conjugated backbone of CN-PPV. Although the efficiency of OSCs based on CN-PPV acceptor is low (efficiencies up to $2 \%$, see Ref. 36), the development of this material triggered efforts in synthesizing polymeric or small-molecular acceptors based on functional groups with strong electron negativity.

In 2015, a high-performing material, with the abbreviated name of ITIC (structure shown in Figure 1e), was developed following these guidelines. ${ }^{37}$ Since then, the best photovoltaic performance for NF OSCs have been obtained with ITIC derivatives (Figure 1f). ${ }^{6,22,38}$ The molecular energy levels and absorption spectra of ITIC derivatives can be effectively tuned by manipulating the intramolecular electron push-pulling effects while keeping their key features as efficient acceptor materials (Figure $1 \mathrm{~g} \mathrm{~h}$ ). Easy tunability makes great contributions to the rapid progress in PCEs for NF OSCs. For example, as shown in Figure 1h, the lowest unoccupied molecular orbital (LUMO) level can be increased by incorporating electron-rich groups like methyl or methoxy into the end-capping units of ITIC, resulting a molecule called IT-M. When mixed with the same polymer, the IT-M-based device have a higher open-circuit voltage $\left(V_{O C}\right)$ value than the ITIC-based device, resulting in an enhanced PCE of $12.1 \%{ }^{38} \mathrm{At}$ the same time, the LUMO level of ITIC could also be reduced by incorporating electrondeficient elements like F-atoms into the end capping groups, resulting in a molecule called IT4F. Since the electron pulling effect of the end groups and hence the intra-molecular charge transfer (ICT) effects are enhanced in IT-4F, this molecule shows a low LUMO level, redshifted absorption and strong extinction coefficient. In order to make good use of the broad and strong light absorption capability of IT-4F without sacrificing the $V_{O C}$, a polymer named PBDBT-SF (shown in Figure 1f) with a low LUMO level was employed as the donor material. As a result, the device based on PBDB-T-SF:IT-4F gave a high efficiency of $13.1 \%$ with a shortcircuit current $\left(J_{S C}\right)$ of $20.5 \mathrm{~mA} / \mathrm{cm}^{2} .6$

A critical question to the OSC materials community is what makes ITIC derivatives so 
unique, in addition to their low bandgap and strong electron accepting capability? We believe that the key might lie in the favorable $\pi-\pi$ interactions enabled by the molecular geometry (Figure 2). In ITIC derivatives, it is the electron-deficient end-capping units that form $\pi$ - $\pi$ interactions with the polymer donors and/or the adjacent acceptor molecules in blend film, facilitating efficient electron transfer and transport. Instead, the electron-rich central unit is not involved in $\pi-\pi$ interactions due to the steric hindrance of the non-conjugated side groups. ${ }^{39} \mathrm{We}$ notice that this feature is also applicable to other high-efficiency acceptors based on strong intramolecular electron push-pulling effects. For example, a small molecular acceptor, namely EH-IDTBR, ${ }^{40}$ though different from ITIC derivatives in chemical structures, also has electrondeficient end-capping units for $\pi-\pi$ interactions and an electron-rich core whose $\pi$ orbitals are shielded by non-conjugated side groups.

\section{Efficient charge separation upon small driving energies}

Compared with fullerene-based OSCs, the most striking feature of NF-based OSCs is that a wide range of devices show efficient charge generation upon small (even negligible) driving energies. ${ }^{9-14}$ Driving energy, traditionally believed to be necessary to split the strongly bound excitons at the D:A interfaces, constitutes an additional voltage loss for OSCs. ${ }^{41-43}$ Previous investigations on fullerene-based OSCs revealed that charge generation could be efficient when the driving energy is decreased to a certain point, below which charge generation usually decreases dramatically, ${ }^{15,16}$ although one exception was also reported. ${ }^{44}$ Therefore, there is usually a trade-off between high photocurrent and small voltage loss for fullerene-based OSCs, limiting the power conversion efficiency. Instead, for NF OSCs, it has been demonstrated that the internal quantum efficiency can approach $90 \%$ even when the driving energy approaches zero, for instance, in the P3TEA:SF-PDI 2 blend. ${ }^{9}$ This is the reason why a range of NF OSCs show high $J_{S C}$ and large $V_{O C}$ at the same time. ${ }^{10-14,45}$ At this stage, it is not clear whether the tolerance with small driving energies comes from some special properties of the NF acceptors or merely from the fact that NF acceptors provide more opportunities to obtain aligned energetic levels between the donor and acceptor materials.

In the context of this Review, the driving energy is defined as the difference between the 
bandgap $\left(E_{g a p}\right)$ of the D/A materials (whichever has a smaller bandgap) and the energy of the charge-transfer (CT) state when the electron-hole pair is still confined to the heterojunction, prior to long range separation. ${ }^{3,15}$ Although it is an important parameter, the $E_{g a p}$ in literature was usually arbitrarily determined from the absorption onset. An appropriate approach might be to use the crossing point between the normalized absorption and luminescence spectra to determine the $E_{\text {gap }} .{ }^{9}{ }^{46}$ This crossing point corresponds to the energy of the transition from the zeroth vibrational ground state to the zeroth vibrational first excited state. An alternative approach suggests the use of the inflection point of the external quantum efficiency (EQE) spectrum at long wavelengths to determine the $E_{\text {gap }} .{ }^{47}$ This method, based only on EQE measurements, might be particularly advantageous for analyzing materials whose absorption onset shifts upon mixing and also enables the determination of the $E_{g a p}$ from materials in literature reports. The CT energies can be determined by measuring the absorption and/or emission from the CT states by using highly sensitive photothermal deflection spectroscopy, Fourier-transform photocurrent spectroscopy - EQE (FTPS-EQE) or electroluminescence (EL) measurements. ${ }^{48-50}$ For systems with large driving energies, a red-shift in the absorption and emission spectra of CT states will be observed compared with those of the singlet excitons from the pristine donor or acceptor materials. ${ }^{15}$ In contrast, for the systems with negligible driving energies, the absorption and emission of the devices will be dominated by those from the pristine donor or acceptor materials (Figure 3a). ${ }^{9,46}$

Note that a small driving energy approaching zero does not necessarily guarantee a small voltage loss, as the voltage losses in OSCs includes the contributions from both the loss due to charge transfer (i.e. the driving energy) and the loss due to non-radiative recombination. More specifically, the voltage losses can be categorized into three contributions (Figure $3 b$ ): ${ }^{9}$

$$
\begin{aligned}
& q \Delta V=E_{\text {gap }}-q V_{O C} \\
& =\left(E_{\text {gap }}-q V_{O C}^{S Q}\right)+\left(q V_{O C}^{S Q}-q V_{O C}^{\text {rad }}\right)+\left(q V_{O C}^{\text {rad }}-q V_{O C}\right) \\
& =\left(E_{\text {gap }}-q V_{O C}^{S Q}\right)+q \Delta V_{O C}^{\text {rad,below } g a p}+q \Delta V_{O C}^{\text {non-rad }} \\
& =\Delta E_{1}+\Delta E_{2}+\Delta E_{3}
\end{aligned}
$$


$V_{O C}^{S Q}$ in the equation is the maximum voltage based on the Shockley-Queisser limit, where the EQE is assumed to be step-wise, i.e. 1 above the gap and 0 below the gap. ${ }^{51}$ Those who are interested in the details of different terms in Eq. 1 are referred to the SI of Ref. 9. The first term of energy loss in equation $1\left(E_{g a p}-q V_{O C}^{S Q}\right)$ is due to the mismatch between radiation received in a narrow solid angle from the sun and omni-directional radiative recombination originating from the absorption above the bandgap. This loss is unavoidable for any type of solar cells and is typically $0.25 \mathrm{eV}$ or above. ${ }^{52}$ The second term in the equation $\left(q \Delta V_{O C}^{\text {rad,below } g a p}=q V_{O C}^{S Q}-\right.$ $\left.q V_{O C}^{r a d}\right)$ is due to additional radiative recombination from the absorption below the bandgap. For fullerene-based OSCs, the absorption from the CT states (i.e. due to the existence of driving energy) is the main contribution of this term, which can be as large as $0.67 \mathrm{eV}$ in the bench mark poly(3-hexylthiophene): [6,6]-phenyl-C61-butyric acid methyl ester blend. ${ }^{52}$ In the systems with negligible driving energies, CT state absorption becomes invisible, as the CT state energy is close to the singlet exciton energy. In this case, this term is only from the non-ideal absorption edge and can be decreased to $0.07 \mathrm{eV}$ in the P3TEA:SF-PDI 2 blend. ${ }^{9}$ The third loss term $\left(q \Delta V_{O C}^{n o n-r a d}=-k T \ln \left(E Q E_{E L}\right)\right)$ is due to non-radiative recombination, where $E Q E_{E L}$ is electroluminescence quantum efficiency of the solar cell when charge carriers are injected into the device in dark. ${ }^{53}$ Low non-radiative $V O C$ losses are made possible when the EQEEL is enhanced. In other words, a great solar cell also needs to be a great light-emitting diode. ${ }^{54}$ Generally OSCs have much stronger non-radiative $V_{O C}$ losses (in the range of $0.30-0.48 \mathrm{~V}$ ) compared with highly efficient inorganic or perovskite solar cells. ${ }^{49,52}$ From this analysis it becomes obvious that systems with small driving energies can still exhibit large voltage losses due to strong non-radiative recombination.

The origin of strong non-radiative recombination in OSCs has puzzled the community for almost one decade. A recent study shows that non-radiative recombination increased with decreasing CT energy of the blends. ${ }^{55}$ The results were interpreted based on the 'energy-gap law' of non-radiative voltage losses, which were assigned to intramolecular vibrations of the organic semiconductor material itself. This model implied that non-radiative recombination is intrinsic to organic semiconductors which have high vibrational carbon-carbon frequency 
modes. However, while Ref. 55 studied a large number of systems, the majority of these systems employed fullerene derivatives as the acceptor, with significant driving energy for charge transfer. The nature of the donor-fullerene $\pi-\pi$ intermolecular interactions may limit the radiative emission rates for these materials systems, and it is not a priori clear whether the conclusions of Ref. 55 generalize to non-fullerene OSCs, especially those with negligible driving energies.

A recent paper investigated non-radiative recombination in non-fullerene OSCs with negligible driving energies. ${ }^{9}$ The FTPS-EQE and EL spectrum of the blend device overlapped with those of the device based on the pristine donor material, indicating that the emission might be from singlet excitons, rather than CT states (Figure 3a). In addition, the non-radiative recombination voltage loss of the optimised device approaches that of device based on the pristine donor material. These results imply that the non-radiative recombination is possibly determined by the emission properties of pristine material (whichever has lower bandgap) in the combinations with negligible driving energies. In other words, it means that it is important to enhance the photoluminescence quantum efficiency of the low bandgap materials to decrease the non-radiative recombination loss in the devices with negligible driving energies. In an ideal case, no photoluminescence quenching of the low bandgap material should be observed upon mixing the donor and acceptor at the $V_{O C}$ so that the photovoltage can be maximised, while the photoluminescence should be completely quenched upon small internal field and at the $J_{S C}$ so that the fill factor (FF) and photocurrent can be maximised. However, since only one combination was investigated in Ref. 9, it is difficult to draw any solid conclusion, especially considering that the observation is purely experimental, without any mechanistic understanding. Further studies combining experimental and theoretical investigations, especially on the systems with negligible driving energies, might shed more light on the origins of non-radiative recombination loss in OSCs. Since the voltage loss due to charge transfer is minimised in the systems with negligible driving energies, a thorough understanding of nonradiative recombination is key to further enhancing the $V_{O C}$ and hence the PCE of OSCs.

Spin statistics might be also important for understanding the nature of the non-radiative non-geminate recombination mechanisms. Following the very well understood issues of the 
spin statistics for electron-hole capture in organic light-emitting diodes $(75 \%$ of these events form triplet excitons that are not emissive and often too low in energy to return to the emissive singlet state), the same spin statistics are expected for non-geminate recombination of electron hole pairs in OSCs. Where there is a molecular triplet on either donor or acceptor that lies lower in energy than the CT energy gap, population of this state via the initially-created triplet CT state causes very efficient non-radiative decay. This has been seen for a number of fullerene systems, ${ }^{56}$ though this is avoided under optimum circumstances, where the CT states formed are only weakly bound (as found for systems with well-formed pure fullerene nanostructures that probably confine the electron away from the fullerene cluster surface). ${ }^{57}$ At present, little is known about this triplet recombination channel for NF acceptor systems.

In addition to non-radiative recombination, another key point is the charge generation process in NF OSCs. Compared with the rapid development in materials and devices, photophysical investigations of the charge generation process are currently lagging behind, with few publications available in literature. ${ }^{9,58,59}$ For fullerene-based OSCs, charge generation is a most intensively investigated issue. ${ }^{3,60-64}$ Various publications found that the initial charge transfer process to form the CT excitation is ultrafast, on a timescale of tens of fs, suggesting that the charge separation process might involve delocalization of acceptor and donor states across the heterojunction. ${ }^{65-67}$ The delocalisation could be due to strong electronic coupling or vibronic (vibrational/electronic) coupling or entropy. Subsequent separation of the electronhole pair to beyond the geminate recombination range, considered to be $>5 \mathrm{~nm}$ (Langevin radius), has been measured to be very fast (as short as $40 \mathrm{fs}$ ), ${ }^{3}$ and the presence of fullerene clusters that provide delocalized $\pi *$ electron wavefunctions over many fullerene units was often believed to play a key role for delocalisation or strong coupling. ${ }^{68}$ This provides a rationale for the avoidance of geminate recombination expected to occur. After the early time rapid separation, localization of the charge carrier wavefunction does set in, as the phonon cloud catches up with electrons. ${ }^{3,64,65}$ It would be very interesting to investigate whether similar or fundamentally different mechanisms are contributing to the charge separation process in NF OSCs, using advanced spectroscopic methods like broadband pump-probe spectroscopy, twodimensional electronic spectroscopy, ${ }^{69,70}$ or pump-push-probe spectroscopy. ${ }^{71}$ Based on the 
understanding developed for fullerene acceptors, the key requirement is the availability of charge carrier states (electron and/or holes) that are delocalized over many acceptor/donor molecules, so that long range charge separation can occur before phonon-driven localization sets in.

\section{Opportunities and challenges}

The emergence of these NF acceptors provides a range of opportunities, which are otherwise not possible in fullerene-based OSCs. We now discuss these opportunities along with challenges from the viewpoints of materials and devices.

\section{Material chemistry of photoactive materials}

It might be the most straightforward strategy to enhance both the photocurrent and photovoltage of NF OSCs by tuning the absorption spectra and energetic levels, which are two main advantages of NF OSCs (Figure $1 \mathrm{~g} \mathrm{~h}$ ). In fact, recent rapid progresses in high-performance NF OSCs are mainly dependent on careful tuning of these two parameters. We use the device based on the PBDT-T-SF:IT-4F donor-acceptor system (Figure 1f), with PCE $=13.1 \%$, as an example to demonstrate possible approaches to further enhancing the efficiency. Although this blend shows a broad EQE spectrum with a maximum value of $83 \%$, the EQE values at short wavelength ranging from 400 to $550 \mathrm{~nm}$ is still low due to limited absorption of the polymer donor in this region. In addition, the blend has a bandgap of $1.58 \mathrm{eV}$ with a $V O C$ of $0.88 \mathrm{~V}$, corresponding to a voltage loss of $0.70 \mathrm{eV}$, which is higher than other high-efficiency NF OSCs with low voltage losses. ${ }^{9}$ Therefore, further improvement of the photocurrent of this system may be obtained with optimizing the absorption of the polymer donor in the short wavelength regime, for instance, by either shifting the polymer absorption spectrum to higher energies or by adding conjugated side chains to enhance the absorption of short wavelength regime; further improvement of the photovoltage may be obtained with optimizing the energetic offsets between the donor and acceptor materials, for instance, by downshifting the energetic levels of the donor and/ or upshifting those of the acceptor.

In addition to absorption spectra and energetic levels, morphology is also of critical importance to the device performance. State-of-the-art small molecules are all amorphous and 
also readily miscible with a range of high-performance polymer donors, raising new requirements for polymer donors to form optimized morphology. In this case, an effective approach to control the phase separation morphologies would be to control the aggregation effects of the donor materials. Indeed, all the polymer donors in high-efficiency NF OSCs share a unique aggregation feature in common, i.e. the co-existence of strong aggregation effects and excellent dispersibility in a solvent. ${ }^{72-74}$ The aggregation effects can be examined by temperature-dependent absorption measurements, as shown in Figure 4a with PBDB-T as an example. ${ }^{75}$ When mixed with small molecular acceptors, the aggregation of these donor polymers confines the nucleation and growth of the acceptors, forming phases with high purity and optimized morphology (Figure $4 \mathrm{~b}$ ). The aggregation effects of the polymer donors can be tuned by changing the solvation of the molecules in solvent, for instance, by changing the solvent or by changing flexible side groups of the molecules. We believe that detailed investigations from the polymer physics point of view will help to further understand the process and provide practical guidance for rational design of materials.

Another critical morphology issue for NF OSCs is the molecular orientation at the D:A interfaces. ${ }^{18-21}$ The charge transfer and transport in organic semiconductors occur through the $\mathrm{sp}^{2}$ hybrid $\pi$-orbitals with a preferred direction from the vertical direction relative to their conjugated surfaces. ${ }^{76,77}$ Therefore, the molecular orientations at these interfaces greatly affect the photovoltaic performance of OSCs. For instance, morphological studies of all polymer OSCs implied that the face-to-face packing mode at the D:A interfaces was more favorable for charge generation than the edge-to-face packing mode. ${ }^{18}$ Although molecular orientation is not critical for OSCs based on fullerene derivatives, which have isotropic conjugated structures, the anisotropic conjugated backbones of NF acceptors bring serious challenges to this issue in NF OSCs. While it is fairly straightforward to understand the correlations between the molecular orientation and photovoltaic performance, how to precisely characterize and control the molecular orientations are two challenging topics in the field.

Charge carrier mobility can also significantly affect the device performance by affecting bimolecular recombination. For organic semiconductors, planar conjugated backbones and high crystallinity are beneficial for intra and inter molecular carrier transport, respectively. However, 
to obtain planar conjugated backbones and/ or high crystallinity is very challenging in BHJ OSCs, since a nanoscale interpenetrating network between D:A molecules is required for efficient charge generation. In order to meet this morphological requirement, high mobilities are sacrificed in state-of-the-art acceptor molecules. For example, as discussed previously, twisted backbones are employed in the small molecules based on PDI derivatives, and alkyls with high steric hindrance are employed in the ITIC-like molecules, limiting their electron transport capabilities. As a result, the electron mobilities of the active layers in high-efficiency NF OSCs could only reach the level of $1 \times 10^{-4} \mathrm{~cm}^{2} / \mathrm{s}$, which is one or two orders of magnitude lower than that in fullerene-based OSCs. An important consequence is that the FF in NF OSCs always drops significantly when the active layer thicknesses increase from $100 \mathrm{~nm}$ to over 200 $\mathrm{nm}$, mainly due to enhanced bimolecular recombination in thick films. ${ }^{6}$ In contrast, fullerenebased OSCs can keep high FF with thicknesses up to a few hundreds of nanometers. ${ }^{7,78,79}$ It is critically important to develop new molecular design strategies that can enhance the intermolecular $\pi-\pi$ interactions without causing strong phase separation in blends. This will be very useful to enhance the EQE values by enhancing light absorption in thick films, and also important for making large area solar panels with better reproducibility, which requires thickness tolerance during the solution-processed fabrication.

We have highlighted the opportunities and challenges based on the parameters important for NF OSCs, including absorption spectra, energy levels, morphology (in terms on both aggregation and molecular orientation), and mobilities. We emphasize that the molecular design strategies to optimize one parameter might negatively affect another one, making it challenging to optimize all the parameters at the same time.

\section{Devices}

Tandem solar cells, where two or more sub-cells with complementary absorption bands are internally integrated, provide an effective approach to enhance the light absorption and reduce the thermal losses in single-junction solar cells. ${ }^{80-83}$ The advantages of tandem structures have not been fully explored in fullerene-based OSCs, as they usually demonstrate large voltage losses. In contrast, NF OSCs provide a great opportunity to significantly enhance PCEs of tandem OSCs, made possible by highly tunable absorption as well as the co-existence of low 
voltage losses and efficient charge separation. By carefully tuning the absorption of both front and rear sub-cells, NF-based tandem cells have demonstrated high PCEs of $13.8 \%{ }^{84}$ and more recently over $14 \% .{ }^{85} \mathrm{~A}$ main reason for such a high efficiency is reduced voltage losses, which have the prospect for further improvement. A critical challenge for current tandem NF OSCs is the photocurrent mismatch between the front and rear sub-cells. This challenge can in principle be solved by increasing the thickness of both front and rear sub-cells with optimized bandgaps, so that different portions of the light can be absorbed by respective sub-cells. However, as discussed previously, the efficiencies of thick NF OSCs are currently limited by poor charge transport.

In addition to tandem cells, ternary devices, where the active layer consists of three components, offer another feasible approach to extend the light absorption of the conventional binary OSCs. ${ }^{86,87}$ The emergence of NF OSCs provides a wide range of possibilities for ternary devices: the third component can be a polymer or small molecule donor, ${ }^{88}$ a fullerene-based acceptor, ${ }^{89,90}$ or an NF acceptor (either polymer or small molecule). ${ }^{91}$ In addition to absorption and energy level considerations, critical to ternary devices is to obtain optimized morphology when a third component is added. In cases where the third component is fullerene derivatives, the ratio of fullerene derivatives is usually kept low for good morphology. In order to significantly extend the light absorption, one of the effective approaches might be to use a third component which is similar to an existing component in chemical structures but different in absorption region. For example, a very recent work employed two ITIC-like acceptors (IT-M and IEICO) and demonstrated that these two acceptors are well miscible with each other. ${ }^{91}$ With the weight ratios changing from 1:0 to $0: 1$, the light absorption contributed by these two acceptors can always effectively contribute to the photocurrent generation. In addition, ternary devices have shown improved stability compared with binary devices. ${ }^{23}$ Rich device physics might also be involved in these new ternary devices. For example, it was previously believed that the two donors or two acceptors in ternary devices form alloy, so that the $V_{O C}$ lies between the $V_{O C}$ values of two binary devices. ${ }^{89,92}$ However, recently a ternary device demonstrated a VOC value higher than the $V O C$ values of both binary devices. ${ }^{93}$ This finding not only provides a unique approach to enhance the photovoltage of ternary devices, but also implies new device 
physics for ternary devices.

Other than aiming to absorb much light in tandem and ternary devices, aiming to absorb part of the light intentionally can also be useful for some applications. A typical example is semi-transparent OSCs, ${ }^{94-96}$ which provide unique opportunities for niche applications, for instance for smart windows. Ideal semi-transparent OSCs are supposed to have weak absorption in the visible region and strong absorption in the near infrared region. ${ }^{97}$ In addition, a low voltage loss is also required for high efficiency. Fullerene-based OSCs can meet neither the absorption nor the small voltages loss requirement. Instead, NF OSCs provide the possibilities to meet both requirements. For example, a low bandgap NF-acceptor, namely IEICO-4F, demonstrates great potential for this application. ${ }^{88}$ Absorption of IEICO-4F mainly locates in the deep-red and near infrared region, ranging from $\sim 700 \mathrm{~nm}$ to $\sim 1000 \mathrm{~nm}$. At the same time, it delivers a high $V O C$ larger than $0.7 \mathrm{~V}$, demonstrating a small voltage loss. In addition to the explorations of more NF acceptors for this application, efforts should also be devoted to achieving an optimized trade-off between transparency and conductivity of transparent electrodes.

\section{Outlook}

Previous efficiency predictions of OSCs are based on the assumption that significant driving energies are needed for efficient charge separation. Since NF OSCs have successfully demonstrated high quantum efficiency upon negligible driving energies, we feel it necessary to re-estimate the efficiency (Figure 5a). All the parameters can be empirically estimated other than non-radiative recombination losses, which the community have yet to understand, as discussed previously. The EQE is assumed to be $85 \%$ above the bandgap; the VOC is determined by Equation 1, where $\Delta \mathrm{E} 1$ is calculated based on the bandgap, $\Delta \mathrm{E} 2$ is assumed to be 0.05 due to non-ideal band edge, ${ }^{9}$ and $\Delta \mathrm{E} 3$ (non-radiative recombination losses) is used as the y axis; the FF is based on the well-established empirical relationship ${ }^{98}$

$$
F F=\frac{\gamma_{O C}-\ln \left(\gamma_{O C}+0.72\right)}{\gamma_{O C}+1}
$$

where $\gamma_{O C}=q V_{O C} / n k T, q$ is the elementary charge, $n$ is the diode ideality factor, $k$ is the 
Bolztmann constant, and $T$ is the temperature. Figure $5 \mathrm{~b}$ shows the maximum $\mathrm{FF}\left(\mathrm{FF}_{\max }\right)$ as a function of the $V_{O C}$, indicating that smaller voltage losses (hence larger $V_{O C}$ ) also mean larger possible FF for a given material. In reality, the FF cannot reach the maximum value even for crystalline GaAs and Si solar cells. ${ }^{99}$ We summarize high-FF OSCs (based on both fullerene and NF acceptors) available in literature in the figure. ${ }^{6,38,100,101}$ We find that the values lie within a range of $\mathrm{FF}_{\max }-(0.1 \pm 0.04)$, and an $\mathrm{FF}$ of $\mathrm{FF}_{\max }-0.1$ is used in the efficiency estimation.

As expected, non-radiative recombination $V_{O C}$ losses play a key in determining the efficiency of the OSCs. A high efficiency of $19 \%$ is within reach at wavelengths around $860 \pm$ $60 \mathrm{~nm}$, if the non-radiative recombination $V O C$ loss could be decreased to $\sim 0.21 \mathrm{~V}$. Therefore, understanding and decreasing the non-radiative recombination is key to further enhancing the PCEs of NF OSCs. The non-radiative recombination losses can potentially originate from the materials and energetic levels. ${ }^{9,55}$ In addition to decreasing non-radiative recombination, optimizing the EQE is also important to reach high efficiency. Although a high EQE of $83 \%$ was reported in literature for NF OSCs, such a high value only covers a small wavelength range. ${ }^{6}$ Especially, most of the high-efficiency NF OSCs show low EQE values at short wavelengths due to limited absorption in this region. The light absorption at short wavelengths can be enhanced by tuning the absorption of D/A materials or by employing thick or ternary films.

We realize that the characterization of non-radiative recombination losses is not readily accessible by most research groups, especially those focusing on materials and device development. In order to make it easy for the materials and device groups to identify the potentials of their practical devices, we also estimate the PCE vs. the energy losses, as shown Figure 5c. All the assumptions in this figure are the same as those in Figure 5a, except the $V_{O C}$, which is now determined by difference between the bandgap and the energy losses.

High efficiency prediction, unique features of NF acceptors, and rapid recent advances in this field, make NF OSCs promising as a practically relevant technology. In order to transfer from the lab-scale devices to large-scale modules using low-cost printing techniques, there are several additional issues to be taken care of. Firstly, green solvents are required for processing. 
Currently, the lab-scale high-efficiency NF OSCs are mainly processed from toxic chlorinated solvents (for instance, chloroform or chlorobenzene). Fortunately, compared to fullerene derivatives, NF acceptors show excellent solubility in a larger range of solvents, offering more opportunities to select green solvents. For example, a NF OSC with over 11\% PCE was recently reported by using a mixture of tetrahydrofuran and isopropanol as processing solvents, which are low toxic and also biodegradable. ${ }^{58}$ Secondly, thickness tolerance is preferable for large scale applications. Currently, the efficiency of NF OSCs drops significantly with increasing thickness from 100 to $250 \mathrm{~nm}$, mainly due to decreasing FF. ${ }^{6}$ As discussed previously, future efforts to increase the carrier mobilities might help to solve this problem. Thirdly, detailed investigations on stabilities are needed. ${ }^{23}$ It is well acknowledged that anisotropic NF acceptors with rigid backbones are less mobile than fullerenes in the blend, leading to better morphological stability than fullerene-based OSCs, which have been proved in a few highly efficient NF OSCs. ${ }^{22}$ However, stability of NF OSCs under different environmental factors (for instance, oxygen, moisture, and elevated temperatures) needs to be systematically studied. Fourthly, new donor and acceptor materials need to be developed. State-of-the-art NF OSC donor and acceptor materials are based on complicated synthesis routes, making the cost of NF OSCs high. It will be desirable for future applications to design new materials, which combine low cost and high performance. 


\section{Acknowledgements}

We thank Thomas Kirchartz for insightful discussions. The work was supported by the National Natural Science Foundation of China (Grant Nos. 91633301, 91333204, 51673201, 21325419, 51711530159), Chinese Academy of Sciences (Grant No. XDB12030200), the Swedish Research Council VR (Grant Nos. 2017-00744, 2016-06146), the Swedish Energy Agency Energimyndigheten (2016-010174), the Swedish Government Strategic Research Area in Materials Science on Functional Materials at Linköping University (Faculty Grant No. SFOMat-LiU \#2009-00971), the Engineering and Physical Sciences Research Council in the UK, and the Knut and Alice Wallenberg foundation (KAW) through a Wallenberg Scholar grant to O.I.

\section{Materials \& Correspondence}

Authors to whom correspondence and requests for materials: JH (hjhzlz@iccas.ac.cn) or FG (fenga@ifm.liu.se). 


\section{Figure captions}

Figure 1 State-of-the-art NF acceptors. (a) Key features of successful NF acceptors (PV and CN-PPV are the first NF acceptor materials used in the heterojunction and bulk heterojunction OSCs, respectively): the functional groups, which are linked by high electron negative atoms (e.g. oxygen or nitrogen) and low electron negative atoms (usually carbon) through a conjugated linkage, offer strong electron accepting capabilities. These functional groups are then linked to conjugated backbones. (b) Two typical types of acceptor materials based on aromatic diimide derivatives: dimeric PDIs and polymeric NDIs, where Ar represents the linkers between the units. (c) Performance and molecular structures of NF OSCs using smallmolecule aromatic diimides (SF-PDI $)$ as the acceptor and P3TEA as the donor. (d) Performance and molecular structures of NF OSCs using polymeric aromatic diimides (N2200) as the acceptor and PTzBI as the donor. (e) Molecular structures of eight ITIC-like acceptors with different intra-molecular charge transfer (ICT) effects, with the structures and photovoltaic performance of the combination PBDBT-T-SF: IT-4F shown in (f). The absorption spectra (g) and molecular energy levels (h) of these ITIC-like molecules can be easily tuned by modulating ICT effects. The molecular energy levels were estimated from the electrochemical cyclic voltammetry measurements. 
Figure 2 Unique features of ITIC and its derivatives. (a) The ITIC molecule includes electronpushing and electron-pulling units, where the electron-pushing units are shield by bulky nonconjugated side chains. (b) Top and side views of the optimal geometry of the ITIC molecule. (c) Bimolecular packing mode indicates that the electron-deficient end-capping units form $\pi-\pi$ interactions with the adjacent acceptor molecule for efficient inter-molecular charge transport and with the donor materials for efficient charge transfer. The electron-rich central unit is not involved in $\pi-\pi$ interactions due to the steric hindrance of the non-conjugated side groups, and is only involved in intra-molecular charge transport. (b, c) adapted from Ref. 39, Wiley. 
Figure 3 Energy losses in NF OSCs. (a) The FTPS-EQE and emission spectra of the blend overlap with those of the pristine devices when the driving energy between the donor and acceptor materials approaches zero; (b) The energy losses from the $E_{g a p}$ to the $q V o c$. The value ranges reported in literature are indicated in the figure. $\triangle \mathrm{E} 1$ is unavoidable for any solar cells and $\triangle \mathrm{E} 2$ was reported to be as small as $70 \mathrm{meV}$ in the P3TEA-SF-PDI 2 blend, where the driving energies between the donor and acceptor materials approaches zero, leaving $\Delta \mathrm{E} 3$ critically important for further enhancing the VOC of OSCs. (a) adapted from Ref. 9, NPG. 
Figure 4 The aggregation effects of the donor materials (PBDB-T as an example) in highefficiency NF OSCs. (a) Temperature dependent absorption spectra of PBDB-T, which shows strong aggregation effects and good dispersity in dilute solution. The insert shows that the solution is clear and that the temperature-dependent color change is well reversible. (b) The topography (top) and phase (bottom) images of the neat PBDB-T, PBDB-T:ITIC and PBDBT:PC71BM films spin-coated from chlorobenzene solution under ambient temperature. The nano-size aggregations can be clearly observed in the neat film, and the two blend films show very similar phase separation morphologies, indicating that the morphology of the blend films are mainly determined by the aggregation of the polymer. 
Figure 5 New efficiency prediction for OSCs based on NF acceptors. (a) The efficiency prediction for NF OSCs based on the fact that they can work efficiently upon negligible driving energies. This figure highlights the importance of reducing the non-radiative recombination losses to further enhance the PCEs. (b) The empirical relationship between the $V_{O C}$ and the FF, together with the reported high-FF devices, including both inorganic and perovskite solar cells (in red), fullerene OSCs (in black), and NF OSCs (in green). The data are collected from Ref. 6,38,99-101. (c) An alternative way to predict the efficiency of NF OSCs, showing the efficiency vs. the energy losses. 


\section{References}

1. Halls, J. J. M. et al. Efficient photodiodes from interpenetrating polymer networks. Nature 376, 498-500 (1995).

2. Yu, G., Gao, J., Hummelen, J. C., Wudl, F. \& Heeger, A. J. Polymer Photovoltaic Cells: Enhanced Efficiencies via a Network of Internal Donor-Acceptor Heterojunctions. Science 270, 1789-1791 (1995).

3. Gélinas, S. et al. Ultrafast Long-Range Charge Separation in Organic Semiconductor Photovoltaic Diodes. Science 343, 512-516 (2014).

4. Schmidt-Mende, L. et al. Self-Organized Discotic Liquid Crystals for High-Efficiency Organic Photovoltaics. Science 293, 1119-1122 (2001).

5. McNeill, C. R. \& Greenham, N. C. Conjugated-Polymer Blends for Optoelectronics. Adv. Mater. 21, $3840-3850$ (2009).

6. Zhao, W. et al. Molecular Optimization Enables over 13\% Efficiency in Organic Solar Cells. J. Am. Chem. Soc. 139, 7148-7151 (2017).

7. Zhao, J. et al. Efficient organic solar cells processed from hydrocarbon solvents. Nat. Energy 1, nenergy201527 (2016).

8. Zhang, S., Ye, L. \& Hou, J. Breaking the 10\% Efficiency Barrier in Organic Photovoltaics: Morphology and Device Optimization of Well-Known PBDTTT Polymers. Adv. Energy Mater. 6, 1502529 (2016).

9. Liu, J. et al. Fast charge separation in a non-fullerene organic solar cell with a small driving force. Nat. Energy 1, nenergy201689 (2016).

10.Cheng, P. et al. Realizing Small Energy Loss of $0.55 \mathrm{eV}$, High Open-Circuit Voltage $>1 \mathrm{~V}$ and High Efficiency $>10 \%$ in Fullerene-Free Polymer Solar Cells via Energy Driver. Adv. Mater. 29, 1605216 (2017).

11.Chen, S. et al. A Wide-Bandgap Donor Polymer for Highly Efficient Non-fullerene Organic Solar Cells 
with a Small Voltage Loss. J. Am. Chem. Soc. 139, 6298-6301 (2017).

12.Baran, D. et al. Reduced voltage losses yield $10 \%$ efficient fullerene free organic solar cells with $>1 \mathrm{~V}$ open circuit voltages. Energy Environ. Sci. 9, 3783-3793 (2016).

13.Bin, H. et al. $11.4 \%$ Efficiency non-fullerene polymer solar cells with trialkylsilyl substituted 2Dconjugated polymer as donor. Nat. Commun. 7, ncomms 13651 (2016).

14.Li, Y. et al. Non-fullerene acceptor with low energy loss and high external quantum efficiency: towards high performance polymer solar cells. J. Mater. Chem. A 4, 5890-5897 (2016).

15.Vandewal, K. et al. Quantification of Quantum Efficiency and Energy Losses in Low Bandgap Polymer:Fullerene Solar Cells with High Open-Circuit Voltage. Adv. Funct. Mater. 22, 3480-3490 (2012). 16.Li, W., Hendriks, K. H., Furlan, A., Wienk, M. M. \& Janssen, R. A. J. High Quantum Efficiencies in Polymer Solar Cells at Energy Losses below 0.6 eV. J. Am. Chem. Soc. 137, 2231-2234 (2015).

17.Ran, N. A. et al. Impact of interfacial molecular orientation on radiative recombination and charge generation efficiency. Nat. Commun. 8, 79 (2017).

18. Ye, L. et al. Manipulating Aggregation and Molecular Orientation in All-Polymer Photovoltaic Cells. Adv. Mater. 27, 6046-6054 (2015).

19.Jung, J. W. et al. Fluoro-Substituted n-Type Conjugated Polymers for Additive-Free All-Polymer Bulk Heterojunction Solar Cells with High Power Conversion Efficiency of 6.71\%. Adv. Mater. 27, 3310-3317 (2015).

20.Lee, J. et al. A Nonfullerene Small Molecule Acceptor with 3D Interlocking Geometry Enabling Efficient Organic Solar Cells. Adv. Mater. 28, 69-76 (2016).

21.Kang, H. et al. From Fullerene-Polymer to All-Polymer Solar Cells: The Importance of Molecular Packing, Orientation, and Morphology Control. Acc. Chem. Res. 49, 2424-2434 (2016). 
22.Zhao, W. et al. Fullerene-Free Polymer Solar Cells with over 11\% Efficiency and Excellent Thermal Stability. Adv. Mater. 28, 4734-4739 (2016).

23.Baran, D. et al. Reducing the efficiency-stability-cost gap of organic photovoltaics with highly efficient and stable small molecule acceptor ternary solar cells. Nat. Mater. 16, 363 (2017).

24.Cnops, K. et al. $8.4 \%$ efficient fullerene-free organic solar cells exploiting long-range exciton energy transfer. Nat. Commun. 5, ncomms4406 (2014).

25.Li, T. et al. Small Molecule Near-Infrared Boron Dipyrromethene Donors for Organic Tandem Solar Cells. J. Am. Chem. Soc. 139, 13636-13639 (2017).

26.Tang, C. W. Two-layer organic photovoltaic cell. Appl. Phys. Lett. 48, 183-185 (1986).

27.Anthony, J. E., Facchetti, A., Heeney, M., Marder, S. R. \& Zhan, X. n-Type Organic Semiconductors in Organic Electronics. Adv. Mater. 22, 3876-3892 (2010).

28.Zhang, X. et al. A Potential Perylene Diimide Dimer-Based Acceptor Material for Highly Efficient Solution-Processed Non-Fullerene Organic Solar Cells with 4.03\% Efficiency. Adv. Mater. 25, 5791-5797 (2013).

29.Lin, Y. et al. A Twisted Dimeric Perylene Diimide Electron Acceptor for Efficient Organic Solar Cells. Adv. Energy Mater. 4, 1400420 (2014).

30.Nielsen, C. B., Holliday, S., Chen, H.-Y., Cryer, S. J. \& McCulloch, I. Non-Fullerene Electron Acceptors for Use in Organic Solar Cells. Acc. Chem. Res. 48, 2803-2812 (2015).

31.Guo, Y. et al. Improved Performance of All-Polymer Solar Cells Enabled by Naphthodiperylenetetraimide-Based Polymer Acceptor. Adv. Mater. 29, 1700309 (2017).

32.Mori, D., Benten, H., Okada, I., Ohkita, H. \& Ito, S. Highly efficient charge-carrier generation and collection in polymer/polymer blend solar cells with a power conversion efficiency of 5.7\%. Energy 
Environ. Sci. 7, 2939-2943 (2014).

33.Li, S. et al. Green-Solvent-Processed All-Polymer Solar Cells Containing a Perylene Diimide-Based Acceptor with an Efficiency over 6.5\%. Adv. Energy Mater. 6, 1501991 (2016).

34.Gao, L. et al. All-Polymer Solar Cells Based on Absorption-Complementary Polymer Donor and Acceptor with High Power Conversion Efficiency of 8.27\%. Adv. Mater. 28, 1884-1890 (2016).

35.Fan, B. et al. Optimisation of processing solvent and molecular weight for the production of greensolvent-processed all-polymer solar cells with a power conversion efficiency over 9\%. Energy Environ. Sci. 10, 1243-1251 (2017).

36.Granström, M. et al. Laminated fabrication of polymeric photovoltaic diodes. Nature 395, 257-260 (1998). 37.Lin, Y. et al. An Electron Acceptor Challenging Fullerenes for Efficient Polymer Solar Cells. Adv. Mater. 27, 1170-1174 (2015).

38.Li, S. et al. Energy-Level Modulation of Small-Molecule Electron Acceptors to Achieve over 12\% Efficiency in Polymer Solar Cells. Adv. Mater. 28, 9423-9429 (2016).

39. Yao, H. et al. Achieving Highly Efficient Nonfullerene Organic Solar Cells with Improved Intermolecular Interaction and Open-Circuit Voltage. Adv. Mater. 29, 1700254 (2017).

40.Cha, H. et al. An Efficient, 'Burn in' Free Organic Solar Cell Employing a Nonfullerene Electron Acceptor. Adv. Mater. 29, 1701156 (2017).

41.Faist, M. A. et al. Competition between the Charge Transfer State and the Singlet States of Donor or Acceptor Limiting the Efficiency in Polymer:Fullerene Solar Cells. J. Am. Chem. Soc. 134, 685-692 (2012).

42.Veldman, D., Meskers, S. C. J. \& Janssen, R. A. J. The Energy of Charge-Transfer States in Electron Donor-Acceptor Blends: Insight into the Energy Losses in Organic Solar Cells. Adv. Funct. Mater. 19, 
1939-1948 (2009).

43.Clarke, T. M. \& Durrant, J. R. Charge Photogeneration in Organic Solar Cells. Chem. Rev. 110, 6736$6767(2010)$.

44.Kawashima, K., Tamai, Y., Ohkita, H., Osaka, I. \& Takimiya, K. High-efficiency polymer solar cells with small photon energy loss. Nat. Commun. 6, ncomms10085 (2015).

45.Li, S. et al. A spirobifluorene and diketopyrrolopyrrole moieties based non-fullerene acceptor for efficient and thermally stable polymer solar cells with high open-circuit voltage. Energy Environ. Sci. 9, 604-610 (2016).

46.Nikolis, V. C. et al. Reducing Voltage Losses in Cascade Organic Solar Cells while Maintaining High External Quantum Efficiencies. Adv. Energy Mater. 7, 1700855 (2017).

47.Rau, U., Blank, B., Müller, T. C. M. \& Kirchartz, T. Efficiency Potential of Photovoltaic Materials and Devices Unveiled by Detailed-Balance Analysis. Phys. Rev. Appl. 7, 044016 (2017).

48.Goris, L. et al. Absorption phenomena in organic thin films for solar cell applications investigated by photothermal deflection spectroscopy. J. Mater. Sci. 40, 1413-1418 (2005).

49.Vandewal, K., Tvingstedt, K., Gadisa, A., Inganäs, O. \& Manca, J. V. On the origin of the open-circuit voltage of polymer-fullerene solar cells. Nat. Mater. 8, 904-909 (2009).

50.Tvingstedt, K. et al. Electroluminescence from Charge Transfer States in Polymer Solar Cells. J. Am. Chem. Soc. 131, 11819-11824 (2009).

51.Shockley, W. \& Queisser, H. J. Detailed Balance Limit of Efficiency of p-n Junction Solar Cells. J. Appl. Phys. 32, 510-519 (1961).

52. Yao, J. et al. Quantifying Losses in Open-Circuit Voltage in Solution-Processable Solar Cells. Phys. Rev. Appl. 4, 014020 (2015). 
53.Ross, R. T. Some Thermodynamics of Photochemical Systems. J. Chem. Phys. 46, 4590-4593 (1967).

54.Miller, O. D., Yablonovitch, E. \& Kurtz, S. R. Strong Internal and External Luminescence as Solar Cells Approach the Shockley \#x2013; Queisser Limit. IEEE J. Photovolt. 2, 303-311 (2012).

55.Benduhn, J. et al. Intrinsic non-radiative voltage losses in fullerene-based organic solar cells. Nat. Energy 2, 17053 (2017).

56.Menke, S. M. et al. Limits for Recombination in a Low Energy Loss Organic Heterojunction. ACS Nano 10, 10736-10744 (2016).

57.Rao, A. et al. The role of spin in the kinetic control of recombination in organic photovoltaics. Nature 500, $435-439$ (2013).

58.Zheng, Z. et al. Efficient Charge Transfer and Fine-Tuned Energy Level Alignment in a THF-Processed Fullerene-Free Organic Solar Cell with 11.3\% Efficiency. Adv. Mater. 29, 1604241 (2017).

59.Tamai, Y. et al. Ultrafast Long-Range Charge Separation in Nonfullerene Organic Solar Cells. ACS Nano (2017). doi:10.1021/acsnano.7b06575

60. Vandewal, K. et al. Efficient charge generation by relaxed charge-transfer states at organic interfaces. Nat.

Mater. 13, 63-68 (2014).

61.Gao, F., Tress, W., Wang, J. \& Inganäs, O. Temperature Dependence of Charge Carrier Generation in Organic Photovoltaics. Phys. Rev. Lett. 114, 128701 (2015).

62.Deibel, C., Strobel, T. \& Dyakonov, V. Role of the Charge Transfer State in Organic Donor-Acceptor Solar Cells. Adv. Mater. 22, 4097-4111 (2010).

63.Brédas, J.-L., Norton, J. E., Cornil, J. \& Coropceanu, V. Molecular Understanding of Organic Solar Cells: The Challenges. Acc. Chem. Res. 42, 1691-1699 (2009).

64.Brédas, J.-L., Sargent, E. H. \& Scholes, G. D. Photovoltaic concepts inspired by coherence effects in 
photosynthetic systems. Nat. Mater. 16, 35-44 (2017).

65.Falke, S. M. et al. Coherent ultrafast charge transfer in an organic photovoltaic blend. Science 344, 10011005 (2014).

66.Grancini, G. et al. Hot exciton dissociation in polymer solar cells. Nat. Mater. 12, 29-33 (2013).

67.Jailaubekov, A. E. et al. Hot charge-transfer excitons set the time limit for charge separation at donor/acceptor interfaces in organic photovoltaics. Nat. Mater. 12, 66-73 (2013).

68.Savoie, B. M. et al. Unequal Partnership: Asymmetric Roles of Polymeric Donor and Fullerene Acceptor in Generating Free Charge. J. Am. Chem. Soc. 136, 2876-2884 (2014).

69.Song, Y., Clafton, S. N., Pensack, R. D., Kee, T. W. \& Scholes, G. D. Vibrational coherence probes the mechanism of ultrafast electron transfer in polymer-fullerene blends. Nat. Commun. 5, 4933 (2014).

70.Bakulin, A. A., Silva, C. \& Vella, E. Ultrafast Spectroscopy with Photocurrent Detection: Watching Excitonic Optoelectronic Systems at Work. J. Phys. Chem. Lett. 7, 250-258 (2016).

71.Bakulin, A. A. et al. The Role of Driving Energy and Delocalized States for Charge Separation in Organic Semiconductors. Science 335, 1340-1344 (2012).

72.Liu, D. et al. Molecular design of a wide-band-gap conjugated polymer for efficient fullerene-free polymer solar cells. Energy Environ. Sci. 10, 546-551 (2017).

73.Zhang, S. et al. A Fluorinated Polythiophene Derivative with Stabilized Backbone Conformation for Highly Efficient Fullerene and Non-Fullerene Polymer Solar Cells. Macromolecules 49, 2993-3000 (2016).

74.Yao, H. et al. A Wide Bandgap Polymer with Strong $\pi-\pi$ Interaction for Efficient Fullerene-Free Polymer Solar Cells. Adv. Energy Mater. 6, 1600742 (2016).

75.Qian, D. et al. Design, Application, and Morphology Study of a New Photovoltaic Polymer with Strong 
Aggregation in Solution State. Macromolecules 45, 9611-9617 (2012).

76.Salleo, A. Charge transport in polymeric transistors. Mater. Today 10, 38-45 (2007).

77.Hutchison, G. R., Ratner, M. A. \& Marks, T. J. Intermolecular Charge Transfer between Heterocyclic Oligomers. Effects of Heteroatom and Molecular Packing on Hopping Transport in Organic Semiconductors. J. Am. Chem. Soc. 127, 16866-16881 (2005).

78.Chen, Z. et al. Low Band-Gap Conjugated Polymers with Strong Interchain Aggregation and Very High Hole Mobility Towards Highly Efficient Thick-Film Polymer Solar Cells. Adv. Mater. 26, 2586-2591 (2014).

79.Zhou, H. et al. Development of Fluorinated Benzothiadiazole as a Structural Unit for a Polymer Solar Cell of 7 \% Efficiency. Angew. Chem. Int. Ed. 50, 2995-2998 (2011).

80.Kim, J. Y. et al. Efficient Tandem Polymer Solar Cells Fabricated by All-Solution Processing. Science 317, 222-225 (2007).

81. You, J. et al. A polymer tandem solar cell with $10.6 \%$ power conversion efficiency. Nat. Commun. $\mathbf{4}, 1446$ (2013).

82.Gilot, J., Wienk, M. M. \& Janssen, R. A. J. Measuring the External Quantum Efficiency of Two-Terminal Polymer Tandem Solar Cells. Adv. Funct. Mater. 20, 3904-3911 (2010).

83.Liu, W. et al. Nonfullerene Tandem Organic Solar Cells with High Open-Circuit Voltage of 1.97 V. Adv. Mater. 28, 9729-9734 (2016).

84.Cui, Y. et al. Fine-Tuned Photoactive and Interconnection Layers for Achieving over 13\% Efficiency in a Fullerene-Free Tandem Organic Solar Cell. J. Am. Chem. Soc. 139, 7302-7309 (2017).

85.Cui, Y., Yao, H., Yang, C., Zhang, S. \& Hou, J. Organic Solar Cells with an Efficiency Approaching 15\%. Acta Polym. Sin. (2017). doi:10.11777/j.issn1000-3304.2018.17297 
86.Zhang, G. et al. High-Performance Ternary Organic Solar Cell Enabled by a Thick Active Layer Containing a Liquid Crystalline Small Molecule Donor. J. Am. Chem. Soc. 139, 2387-2395 (2017).

87.Lu, L., Xu, T., Chen, W., Landry, E. S. \& Yu, L. Ternary blend polymer solar cells with enhanced power conversion efficiency. Nat. Photonics 8, 716-722 (2014).

88. Yao, H. et al. Design, Synthesis, and Photovoltaic Characterization of a Small Molecular Acceptor with an Ultra-Narrow Band Gap. Angew. Chem. Int. Ed. 56, 3045-3049 (2017).

89.Lu, H. et al. Ternary-Blend Polymer Solar Cells Combining Fullerene and Nonfullerene Acceptors to Synergistically Boost the Photovoltaic Performance. Adv. Mater. 28, 9559-9566 (2016).

90.Zhao, W., Li, S., Zhang, S., Liu, X. \& Hou, J. Ternary Polymer Solar Cells based on Two Acceptors and One Donor for Achieving 12.2\% Efficiency. Adv. Mater. 29, 1604059 (2017).

91.Yu, R. et al. Two Well-Miscible Acceptors Work as One for Efficient Fullerene-Free Organic Solar Cells. Adv. Mater. 29, 1700437 (2017).

92.Khlyabich, P. P., Burkhart, B. \& Thompson, B. C. Compositional Dependence of the Open-Circuit Voltage in Ternary Blend Bulk Heterojunction Solar Cells Based on Two Donor Polymers. J. Am. Chem. Soc. 134, 9074-9077 (2012).

93.Wang, C. et al. Ternary organic solar cells with enhanced open circuit voltage. Nano Energy 37, 24-31 (2017).

94.Chen, C.-C. et al. High-performance semi-transparent polymer solar cells possessing tandem structures. Energy Environ. Sci. 6, 2714-2720 (2013).

95.Xu, G. et al. High-Performance Colorful Semitransparent Polymer Solar Cells with Ultrathin HybridMetal Electrodes and Fine-Tuned Dielectric Mirrors. Adv. Funct. Mater. 27, 1605908 (2017).

96.Zhang, M., Guo, X., Ma, W., Ade, H. \& Hou, J. A Large-Bandgap Conjugated Polymer for Versatile 
Photovoltaic Applications with High Performance. Adv. Mater. 27, 4655-4660 (2015).

97.Wang, W. et al. Fused Hexacyclic Nonfullerene Acceptor with Strong Near-Infrared Absorption for Semitransparent Organic Solar Cells with 9.77\% Efficiency. Adv. Mater. 29, 1701308 (2017).

98.Green, M. A. Solar cell fill factors - General graph and empirical expressions. Solid State Electron. 24, 788 (1981).

99.Green, M. A., Emery, K., Hishikawa, Y., Warta, W. \& Dunlop, E. D. Solar cell efficiency tables (version 47). Prog. Photovolt. Res. Appl. 24, 3-11 (2016).

100. Jao, M.-H., Liao, H.-C. \& Su, W.-F. Achieving a high fill factor for organic solar cells. J. Mater. Chem. A 4, 5784-5801 (2016).

101. Li, S. et al. Design of a New Small-Molecule Electron Acceptor Enables Efficient Polymer Solar Cells with High Fill Factor. Adv. Mater. (2017). doi:10.1002/adma.201704051 


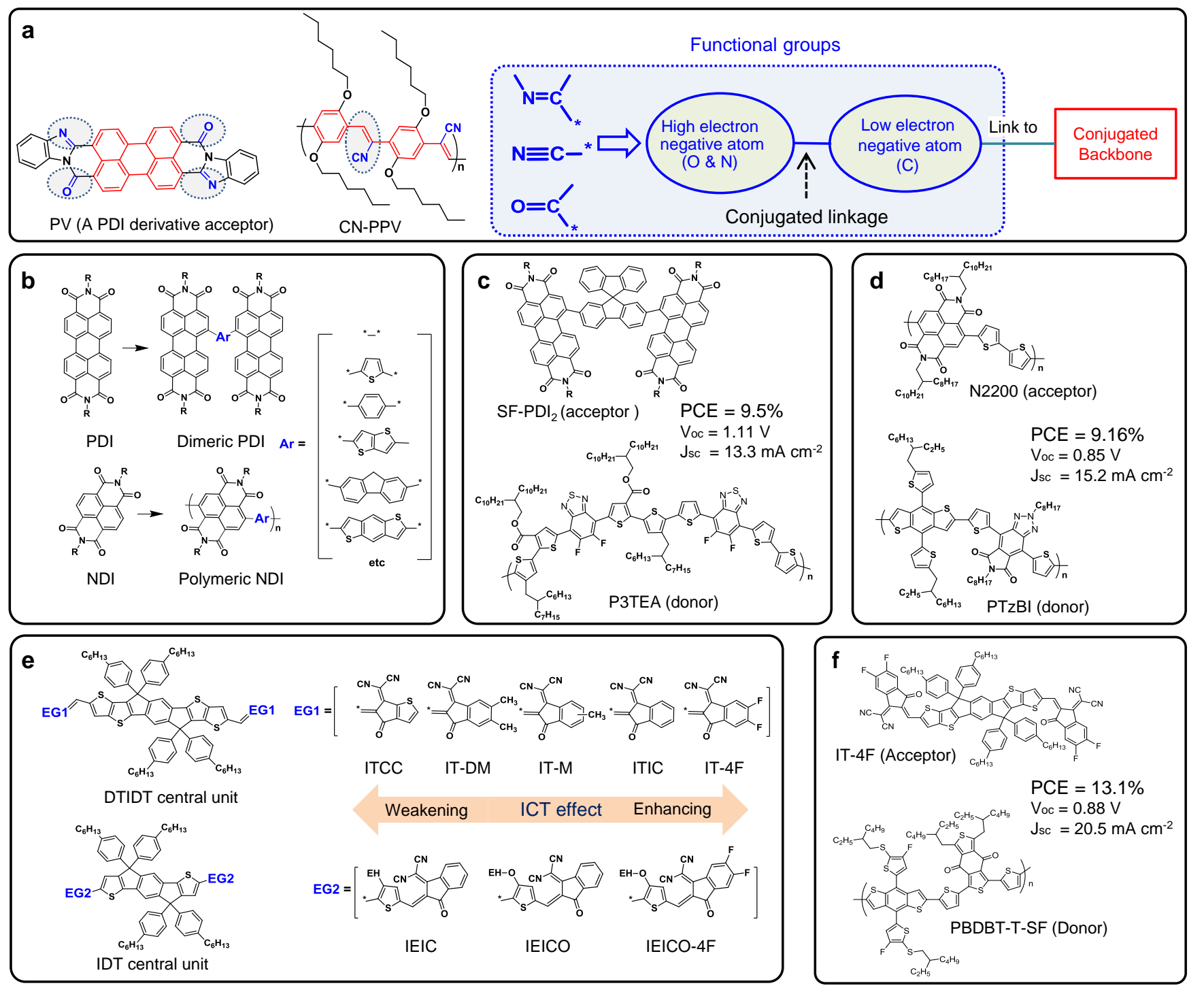

g
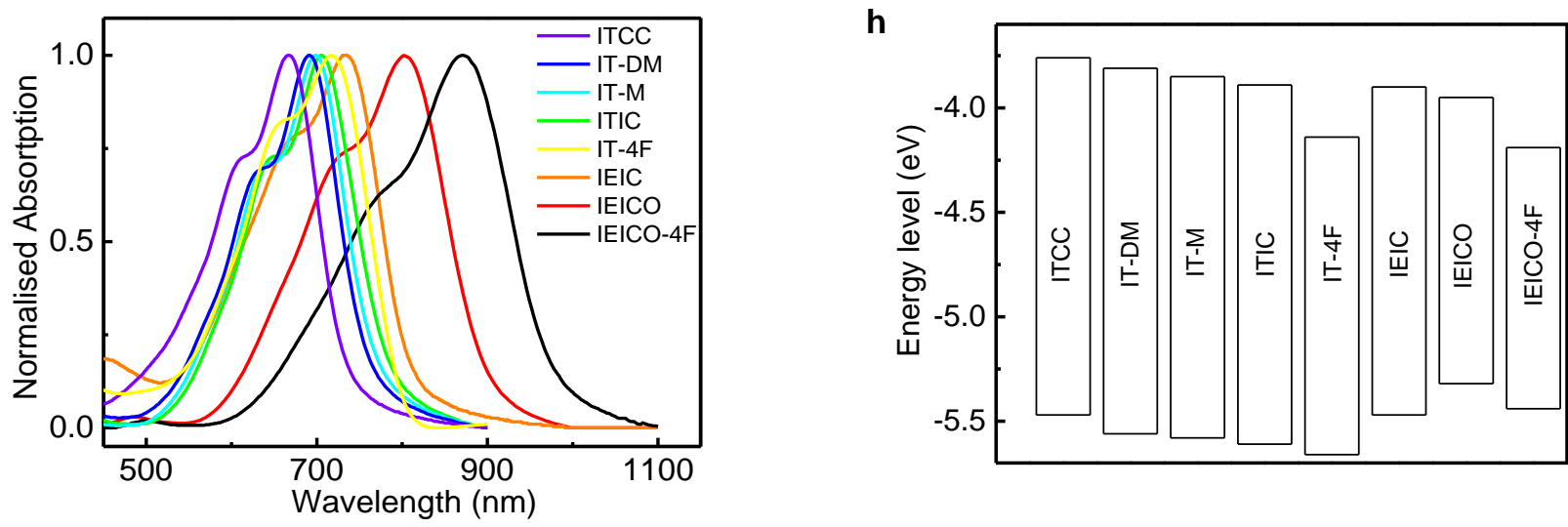

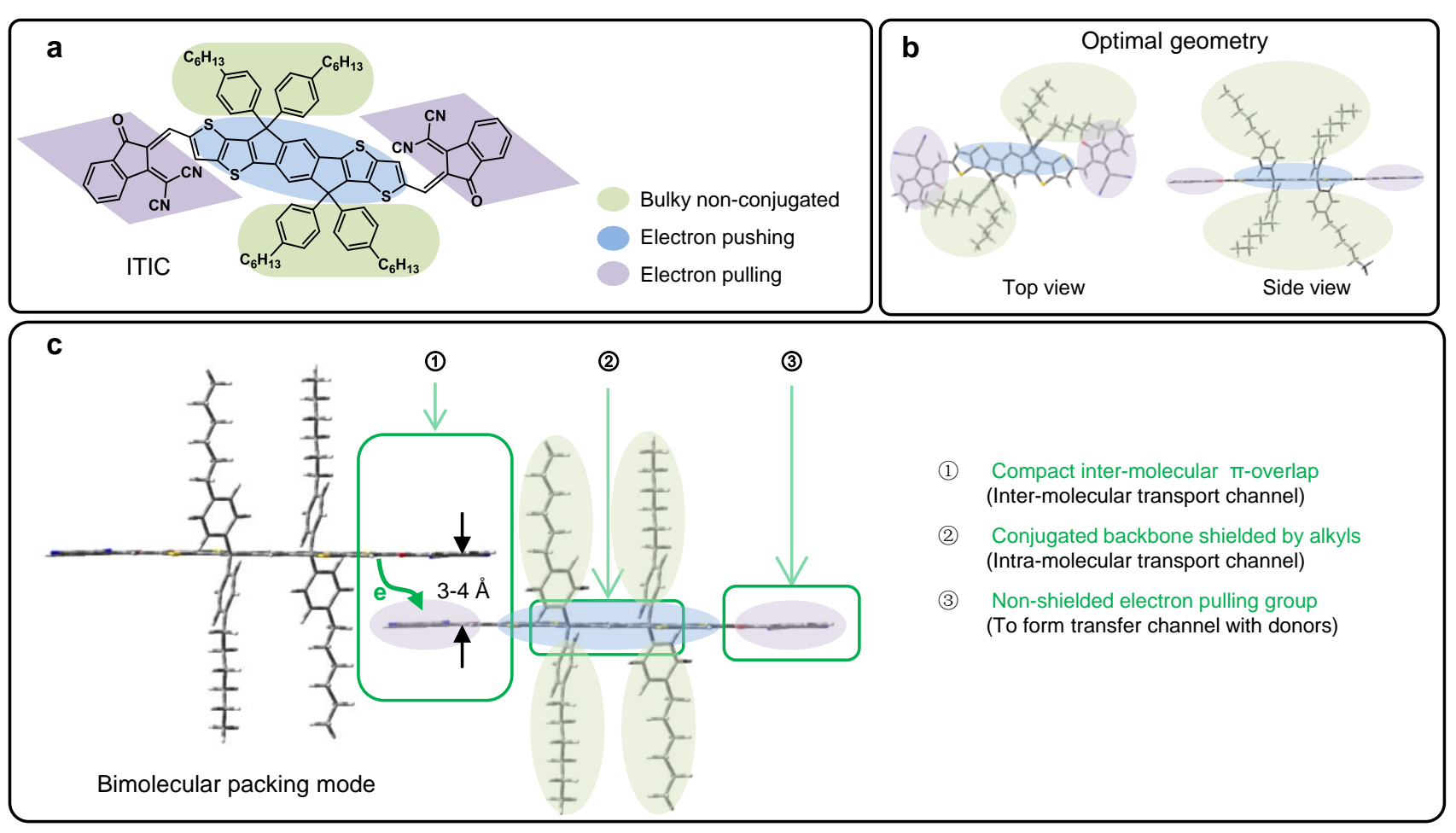
a

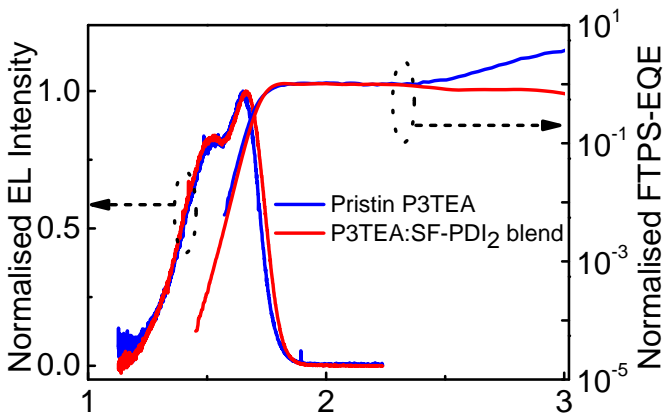

Energy (eV)

b

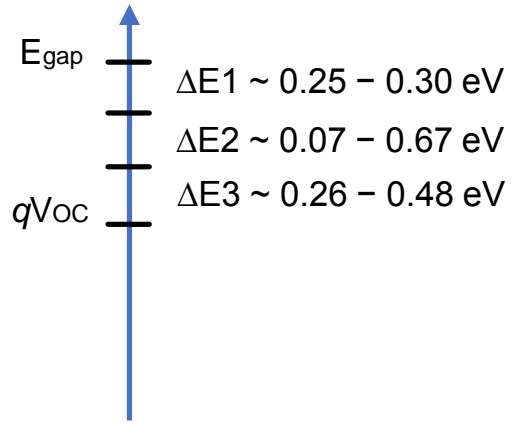


a
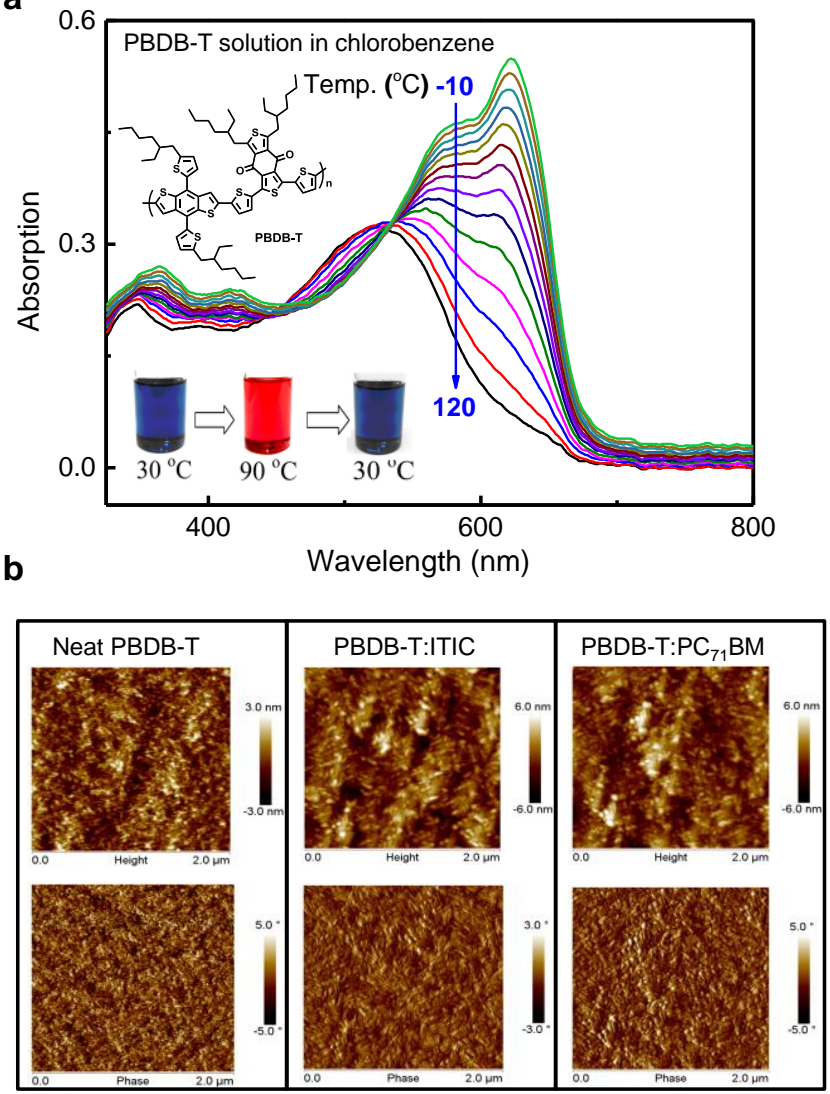
